\title{
Anti-inflammatory combinatorial therapy to enhance killing efficacy with patient- derived preclinical models
}

\author{
Zhang Jing, Bee Luan KHOO* \\ Affiliation : City University of Hong Kong \\ *Contact: blkhoo@cityu.edu.hk, 85234429423
}




\section{Cancer stem cells (CSCs)}

- Drug-resistant cancer phenotypes is a challenge for anti-cancer therapy.

- CSCs are relatively quiescent cells capable of self-renewal.

- CSCs are identified as one of the ways by which chemoresistance develops.

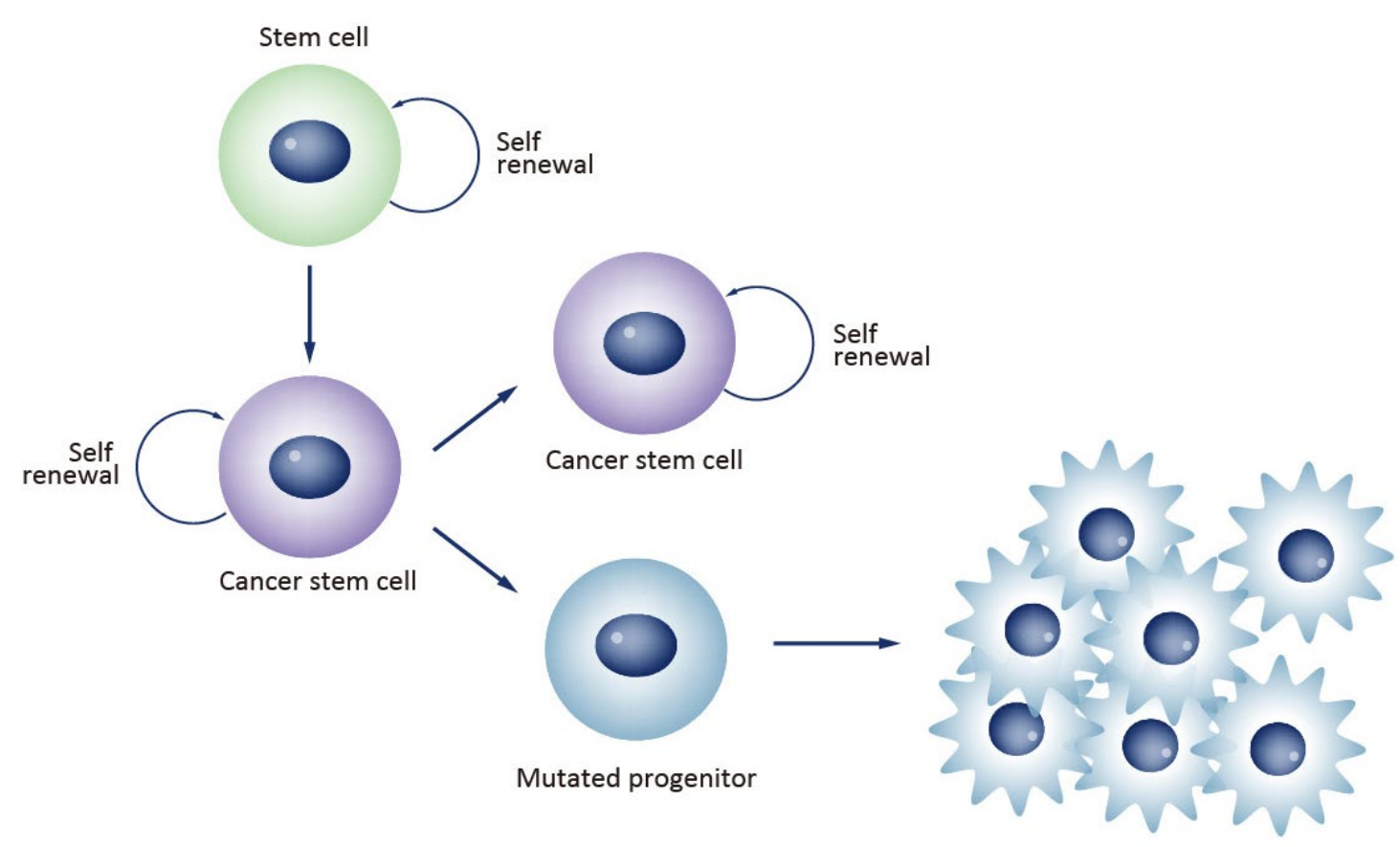




\section{Cancer metastasis}

- Cancer metastasis is the major cause of cancer morbidity and mortality

- accounts for about $\mathbf{9 0 \%}$ of cancer deaths

- a process in which cancer cells disseminate from the primary tumor, settle and grow at a site other than the primary tumor site.
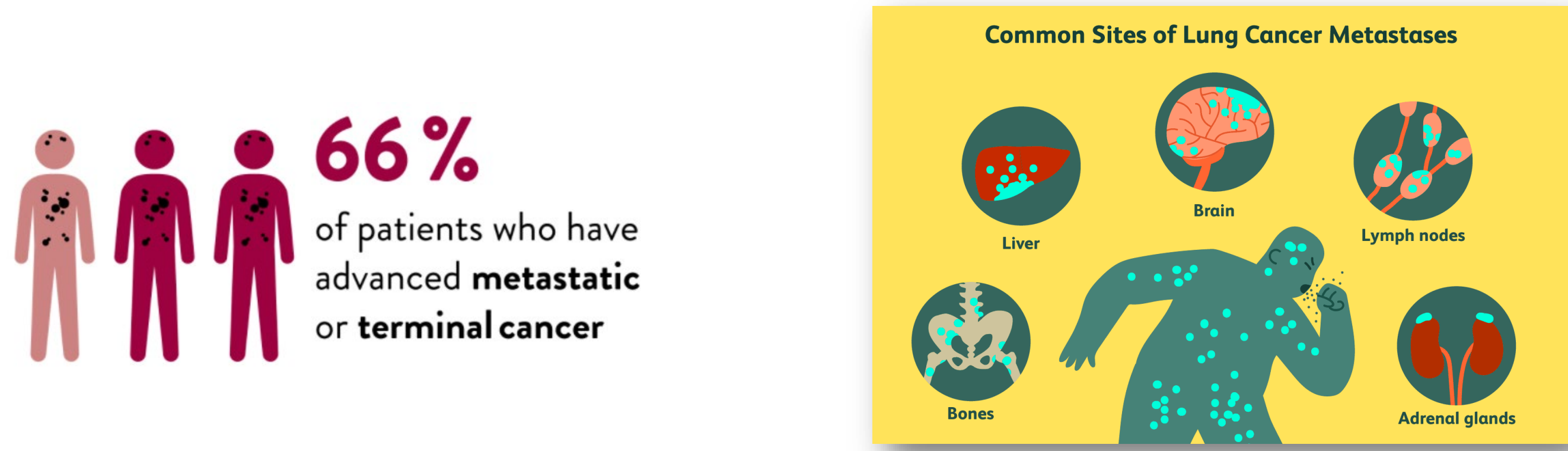


\section{COX-2 and cancer}

- An inhibition of redox-responsive cyclooxygenase (COX) enzymes is often attributed to the mechanism of aspirin.

- COX-2 regulates tumour growth, invasion and metastasis in breast cancer.

- the pro-neoplastic effects of COX-2 action

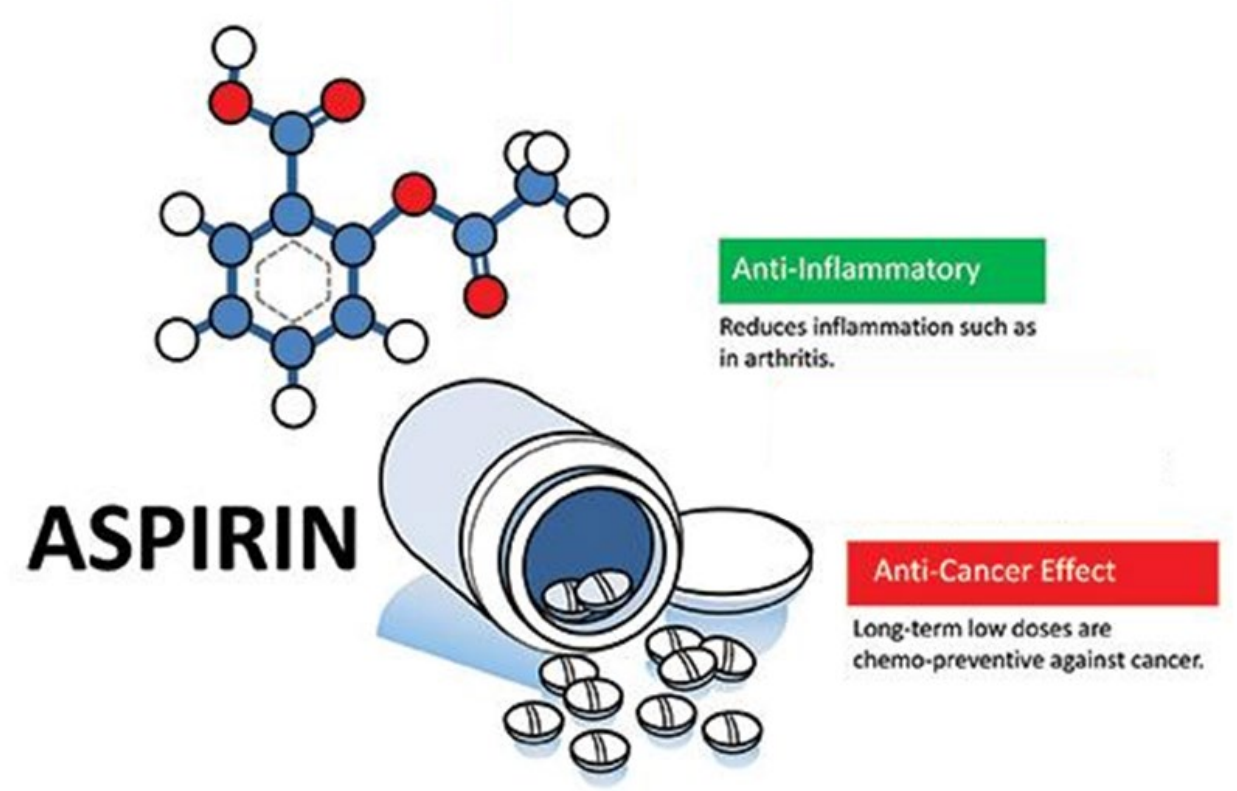




\section{ROS and DNA damage}

- Reactive oxygen species (ROS) are a group of short-lived, highly reactive, oxygencontaining molecules

- induce DNA damage and affect the DNA damage response (DDR).

- COX-2 expression could be triggered by ROS

- ROS favors the expression of an inflammatory phenotype that leads to the induction of COX-2.

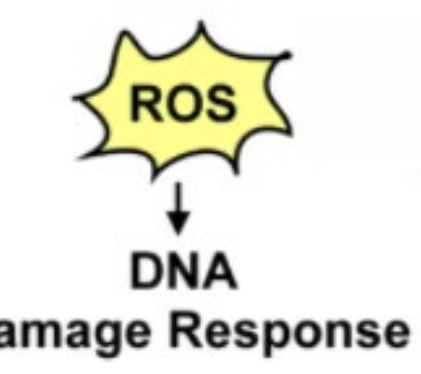




\section{Circulating tumor cells (CTCs) cluster assay}

- CTCs are a rare subset of cells found in the blood of patients with solid tumors.

- We demonstrate an efficient approach to evaluate drug response using patient-derived CTC cultures obtained from liquid biopsy.
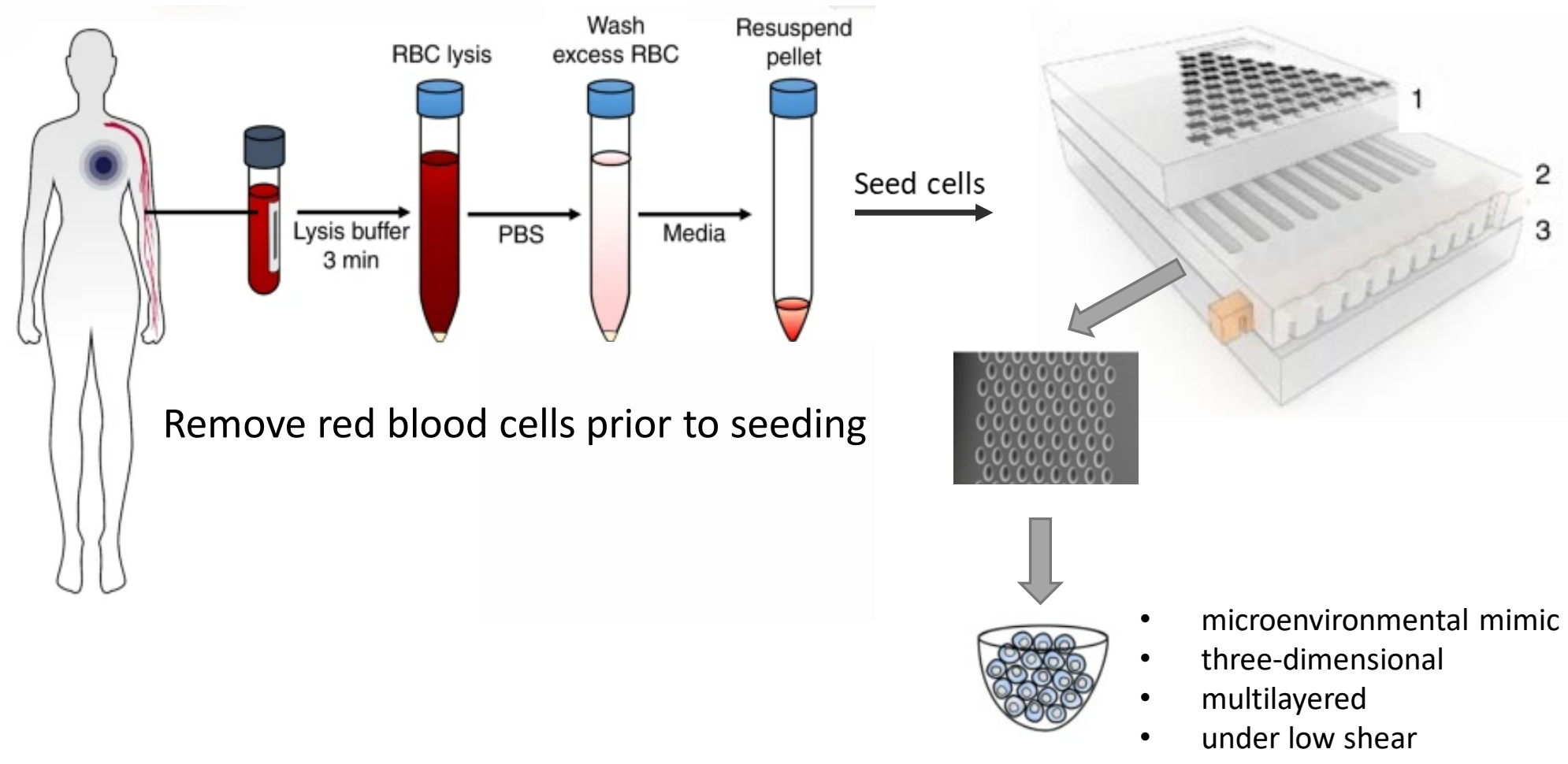

open/closed valve
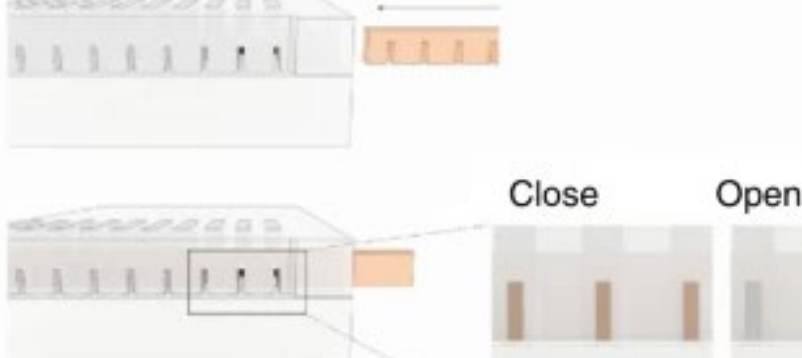

Open 


\section{Combinatorial therapy}

DA treatment: In this proof of concept study, a range of therapeutic drug concentrations for $0-500 \mathrm{mg} / \mathrm{ml}$ aspirin (A) and 0-1 $\mu \mathrm{M}$ doxorubicin (D) were screened with a microfluidic culture and drug-screening assay validated for primary cell cultures.

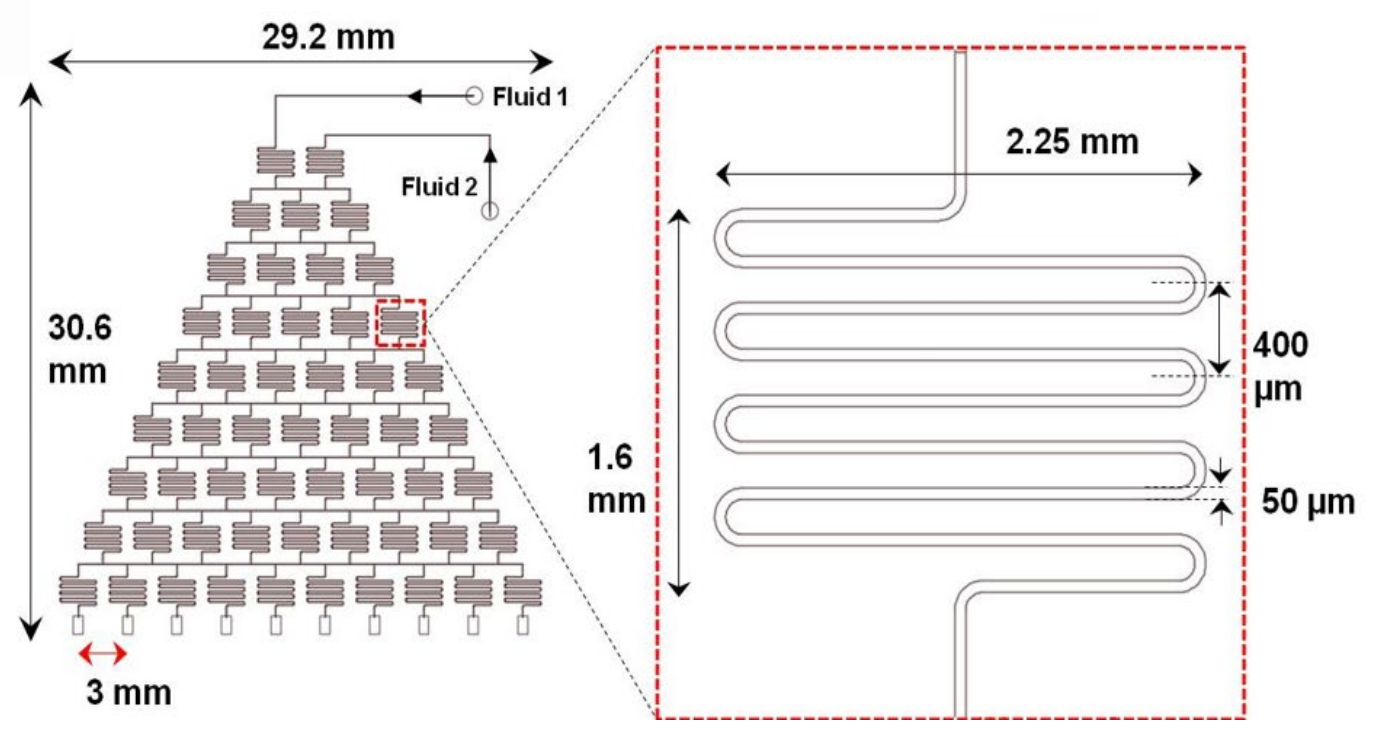

Schematics of the gradient generator

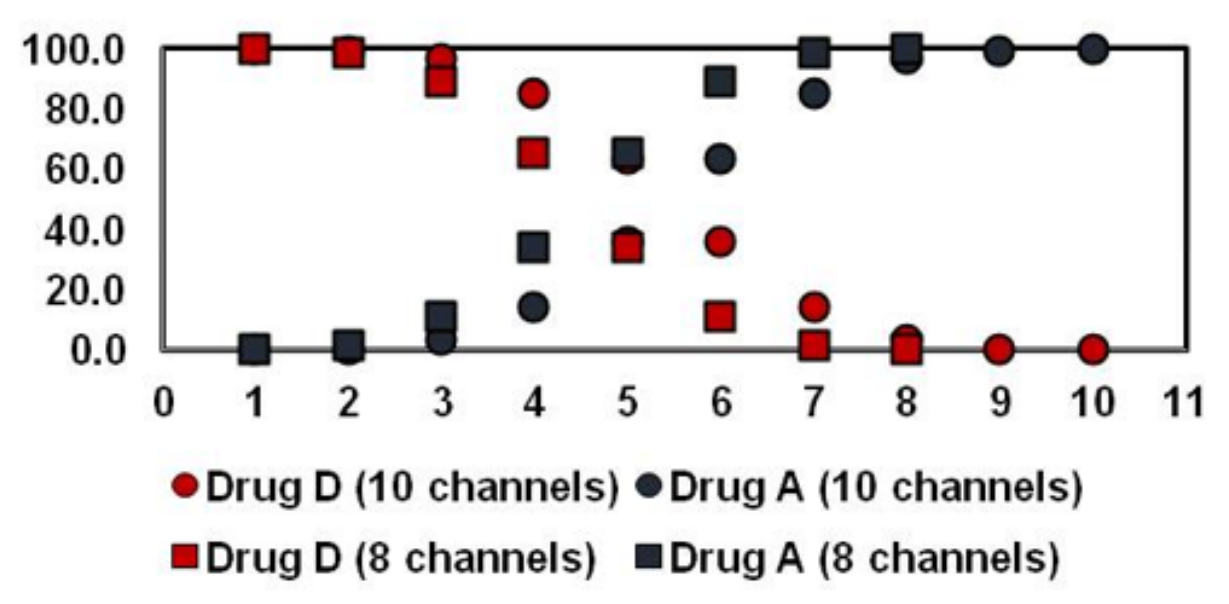

Distribution of two drugs in combination with the effect of gradient generator 


\section{Significance of liquid biopsy and CTCs}

- Cancer is a dynamic disease. During the course of disease, cancers generally become more heterogeneous.

- Heterogeneous response of therapy highlights the importance of patient-derived preclinical models.

- lack of a robust anticancer drug screening system to monitor patients during treatment.

- Our CTC assay obtained from liquid biopsy is efficient, non-invasive, inexpensive and drug screening for personalized treatment.

\begin{tabular}{ll}
\hline Methods & Limitation \\
\hline Imaging techniques & false-negative findings \\
\hline Tumor biopsies & $\begin{array}{l}\text { invasive, cancer gene reflecting } \\
\text { incompletely } \\
\text { Multiwell plates }\end{array}$ \\
\hline Other CTC expansions & pre-enrichment required, low efficiency \\
\hline
\end{tabular}
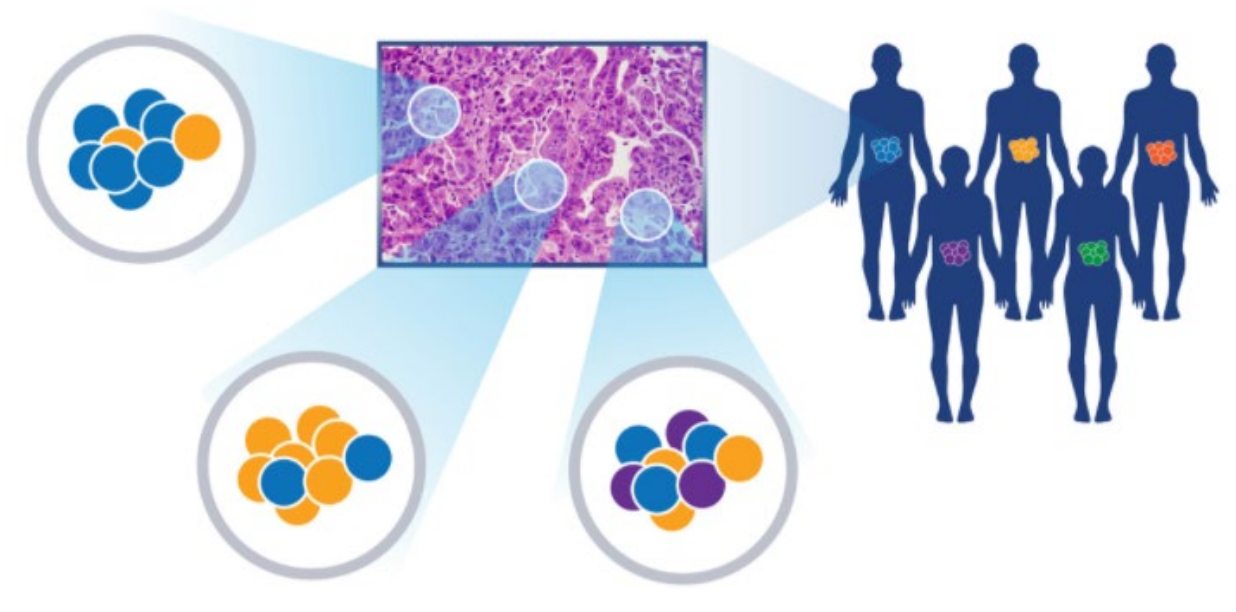

Heterogeneity 


\section{DA therapy improved killing efficacy and apoptosis}

\section{A \\ B}
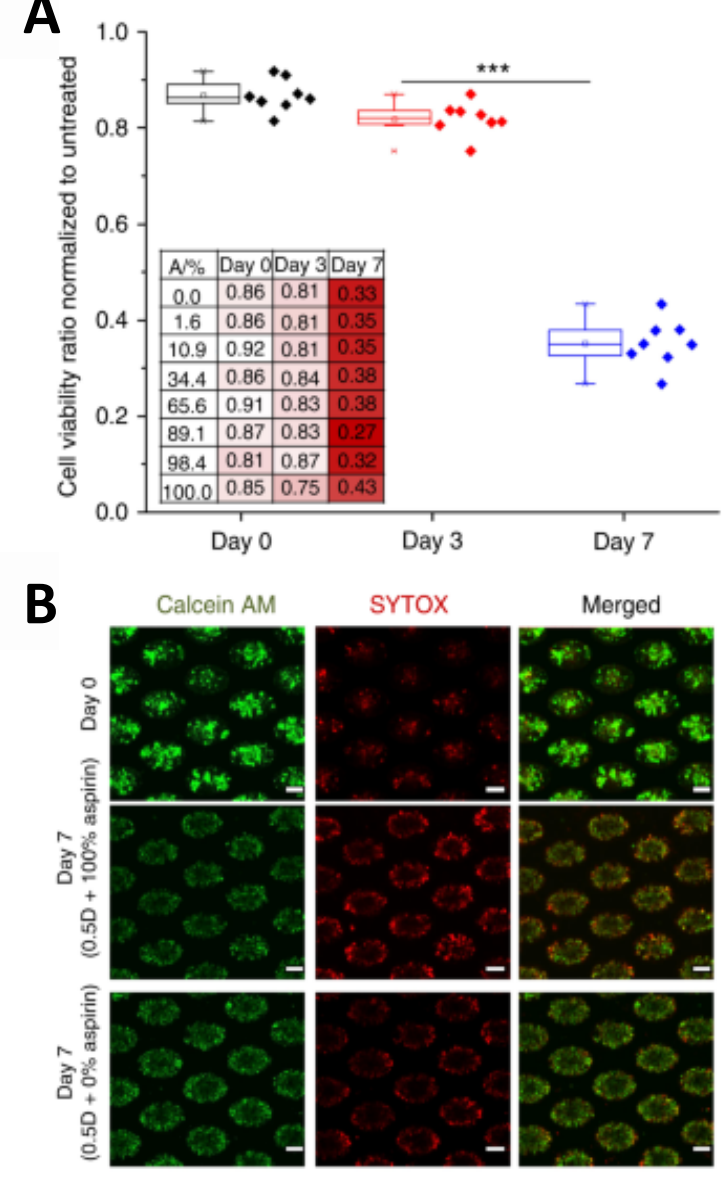

C

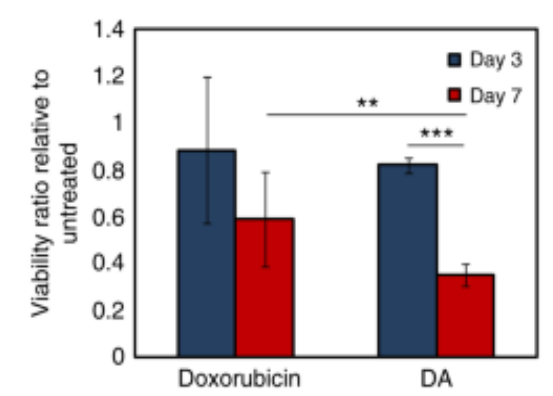

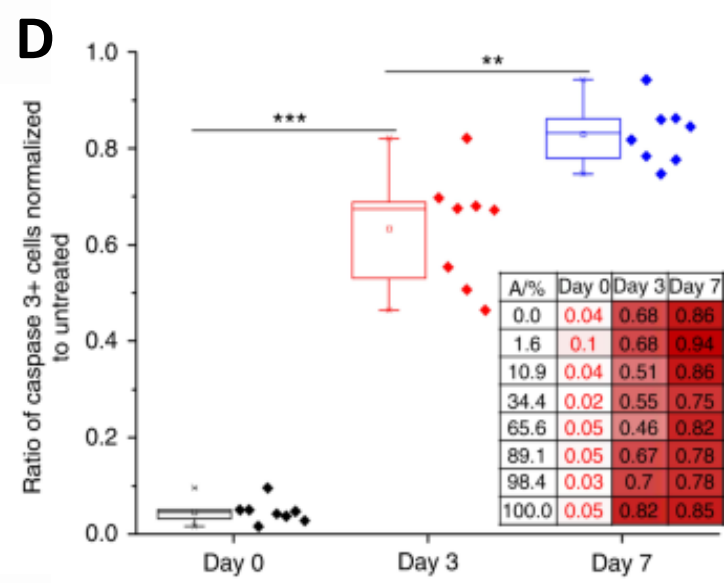

E

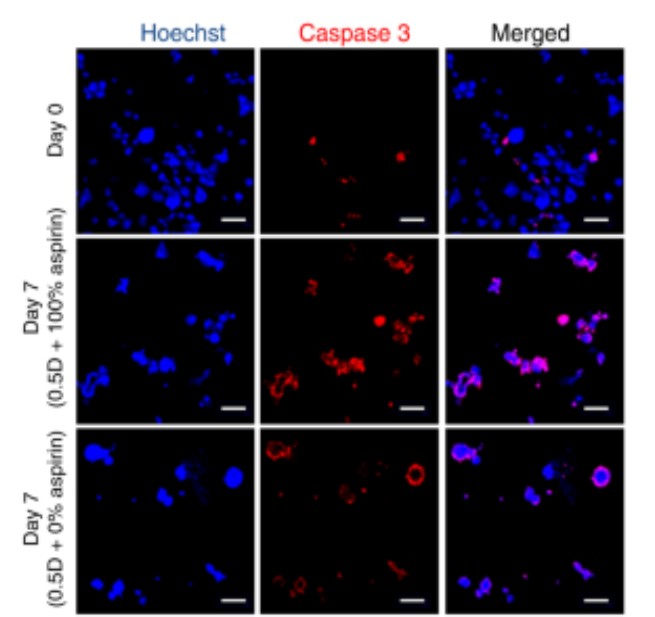

$\mathbf{F}$

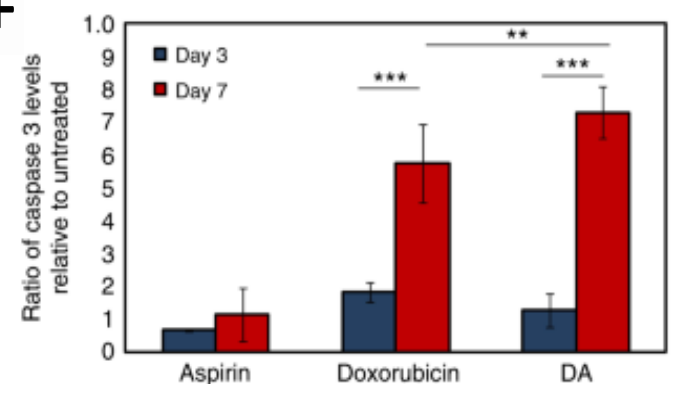

- Viability ratio breast cancer cell line MDA-MB-231 cultures decreases after prolonged exposure to combinatorial DA treatment.

- Killing efficacy of DA treatment surpassed that of treatment with doxorubicin alone after 7 days of exposure, as determined by the live and apoptotic cell proportions. 


\section{DA therapy reduced cancer stem cells}
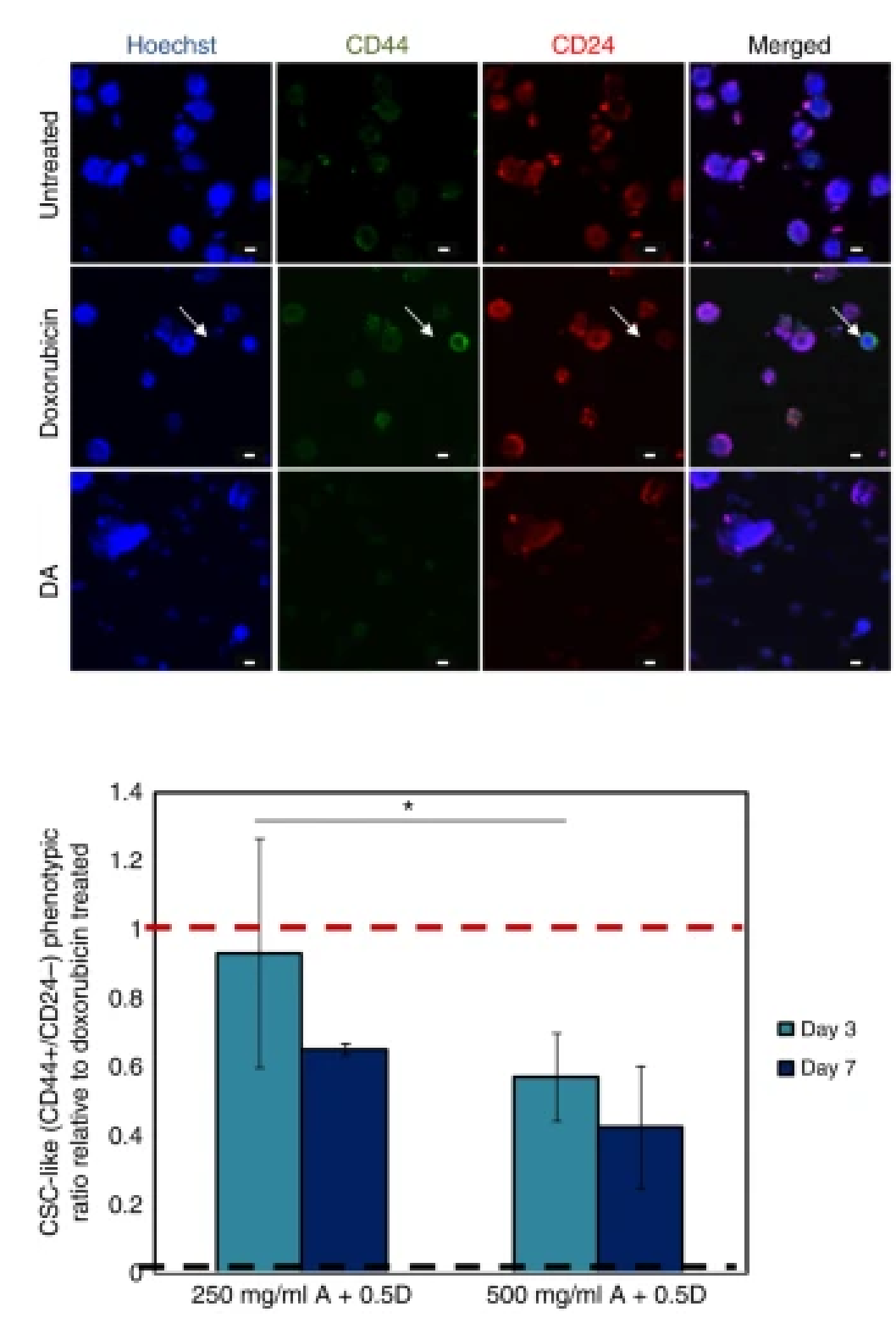

香港城市大學

City University of Hong Kong

- Increase in CSCs post treatment have a high potential to form secondary metastases, leading to cancer relapse.

- $\mathrm{CD}_{4} 4^{+} / \mathrm{CD} 24^{-}$phenotype corresponded to the proportion of CSC-like cells

- DA treatment with $\mathbf{5 0 0} \mathbf{~ m g} / \mathbf{m l}$ aspirin resulted in a significant reduction of CSC proportion. 


\section{DA therapy in clinical cohorts}

- Representative images of microwell array with clusters (left) and without clusters (right). Scale bar is $50 \mu \mathrm{m}$.

- Patient samples that responded in terms of killing efficacy are marked in red.

- Heterogeneity of patient profiles and the reliable patient-derived models are importance for screening similar anti-inflammatory and anticancer strategies
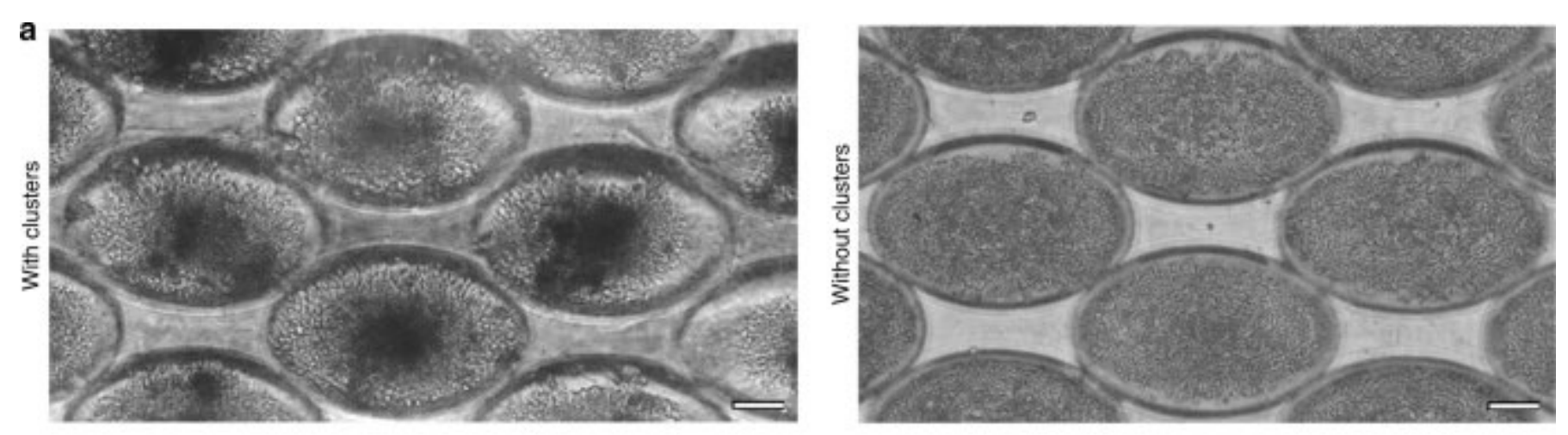

b

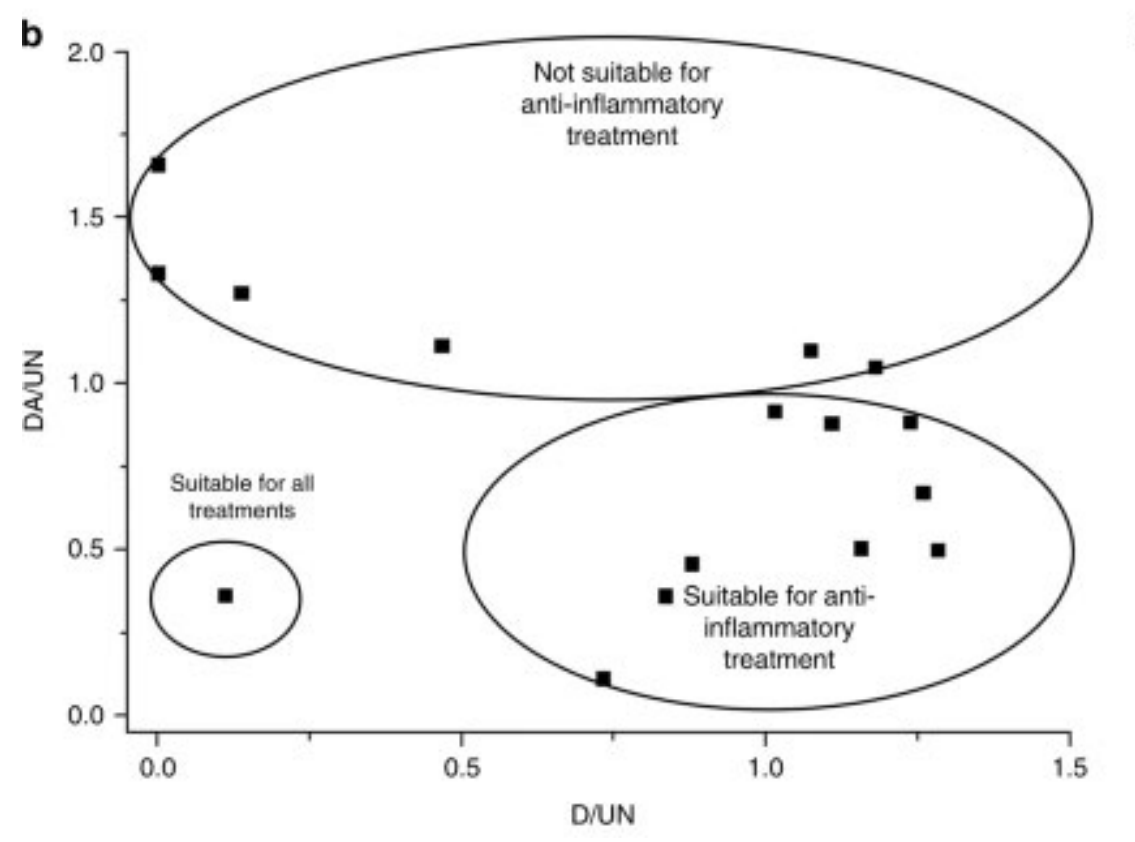

Suitable for antiinflammatory treatment

ASL32 0.39056

BC234 0.15439

$\begin{array}{ll}\mathrm{BC} 249 & 0.43289\end{array}$

BC241 0.4370

BC239 0.52311

CES64 0.71535

CES78 0.78291

CES16 $\quad 0.79557$

CES83 0.8914

BC240 0.90546
Not suitable for anti-inflammatory treatment

CES105 1.02654 CES093_2 1.33 CES76 $\quad 1.66$ $\begin{array}{ll}\text { ASL36 } & 2.39023\end{array}$ CES102 3.27325 CES093_1 9.2865 


\section{DA treatment acts via a COX-independent pathway mediated by anti-inflammatory mechanisms}

- COX-2 protein expression was reduced under combinatorial DA treatment.

- COX-2 reduction is directly associated with malignancy and resistance.

- Reduction in COX-2 protein expression was significantly greater under low-dose aspirin ( $200 \mathrm{mg} / \mathrm{ml})$.

- DA treatment is more effective in its anti-cancer effects.

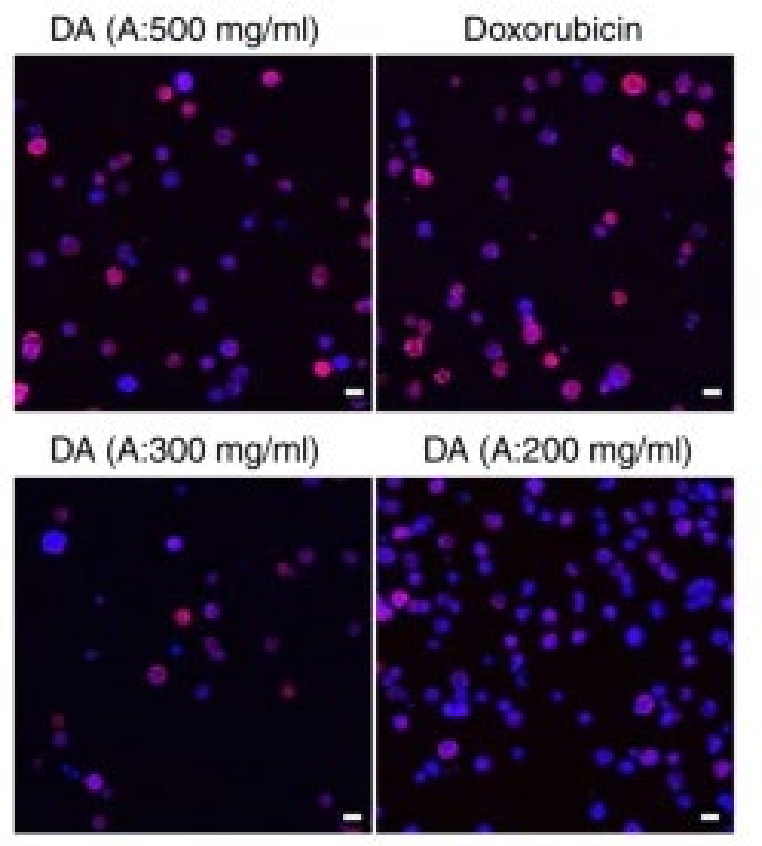

Immunostaining for COX-2 protein. Scale bar is $10 \mu \mathrm{m}$.

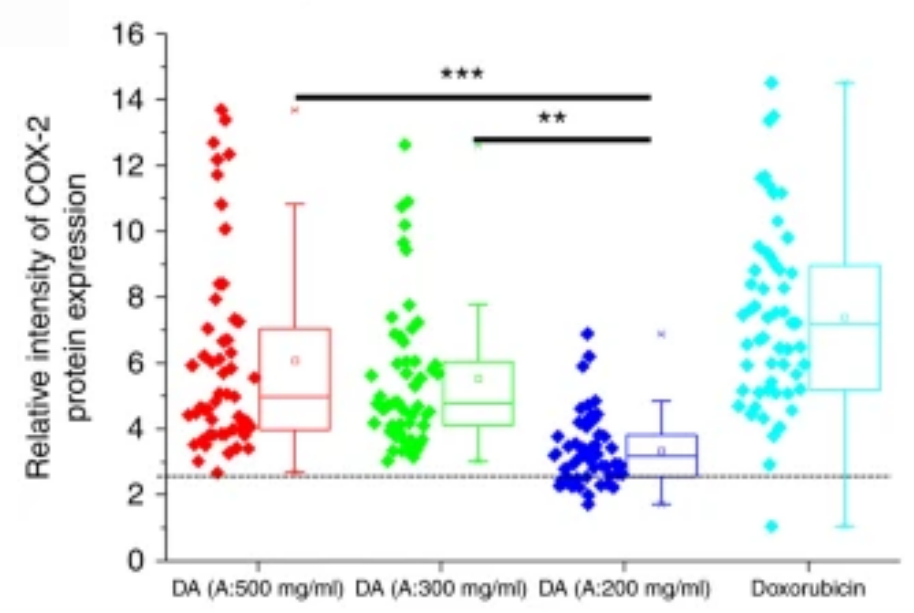

Relative intensity levels of COX-2 protein after 7 days of drug exposure. 


\section{DA treatment reduces intracellular oxidative activity}

- Calcein AM is also an indicator of intracellular oxidative activity.

- The differential intensity of Calcein AM suggests a reduction in intracellular oxidative activity in samples under both single drug aspirin and combinatorial DA therapy.

- Intracellular oxidative activity is a factor in metabolism and a key regulating process for several core functions including cell proliferation and transcription.
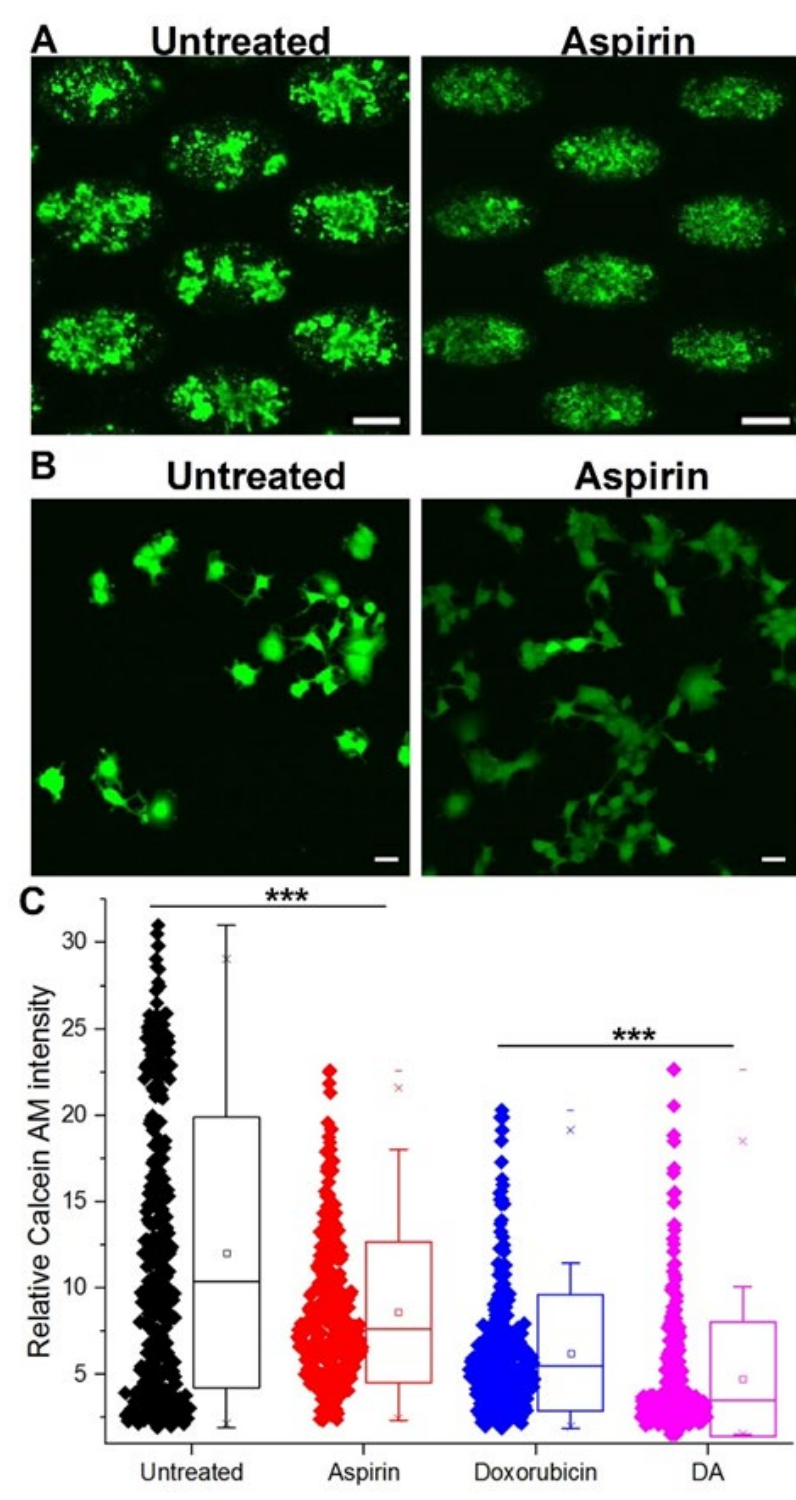

Cluster cultures

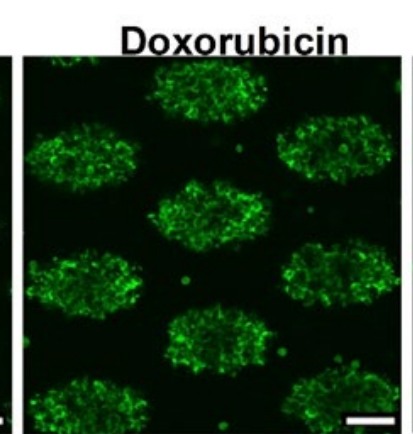

Doxorubicin
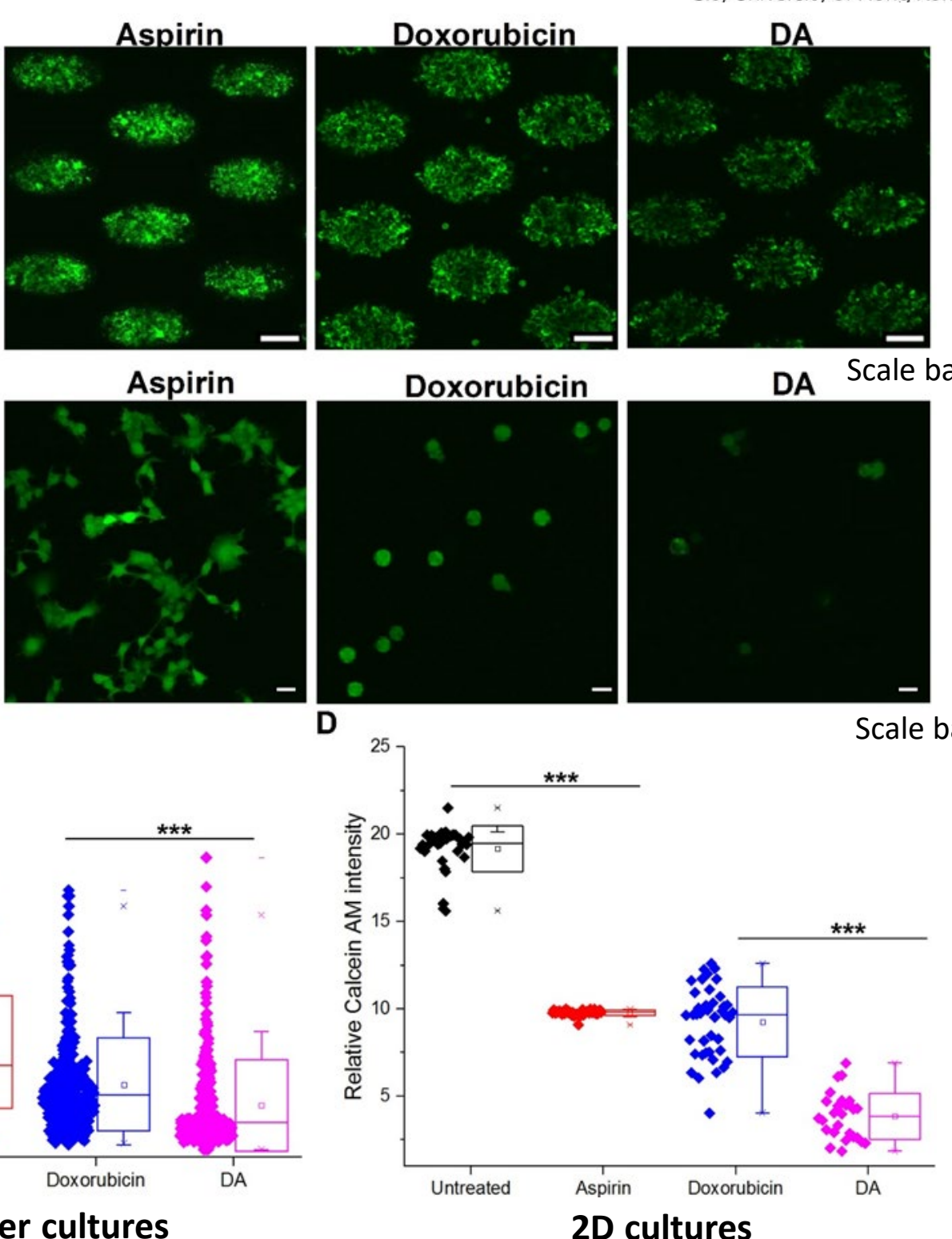

DA Scale bar $100 \mu \mathrm{m}$

bar $20 \mu \mathrm{m}$ 


\section{DA treatment revert cells to a less metastatic phenotype}

- Increased ROS levels causes cancer development and metastasis during or after chemotherapy.

- Extracellular ROS help to facilitate the formation of multifoci tumors and act as a springboard for metastatic tumor cells.

- Cancer cells usually present as a hybrid phenotype and express intermediate levels of epithelial (E) and mesenchymal (M) characteristics.

- With DA treatment, the proportion of cells with epithelial-like phenotypes were increased within $72 \mathrm{~h}$ and more apparent after 7 days.
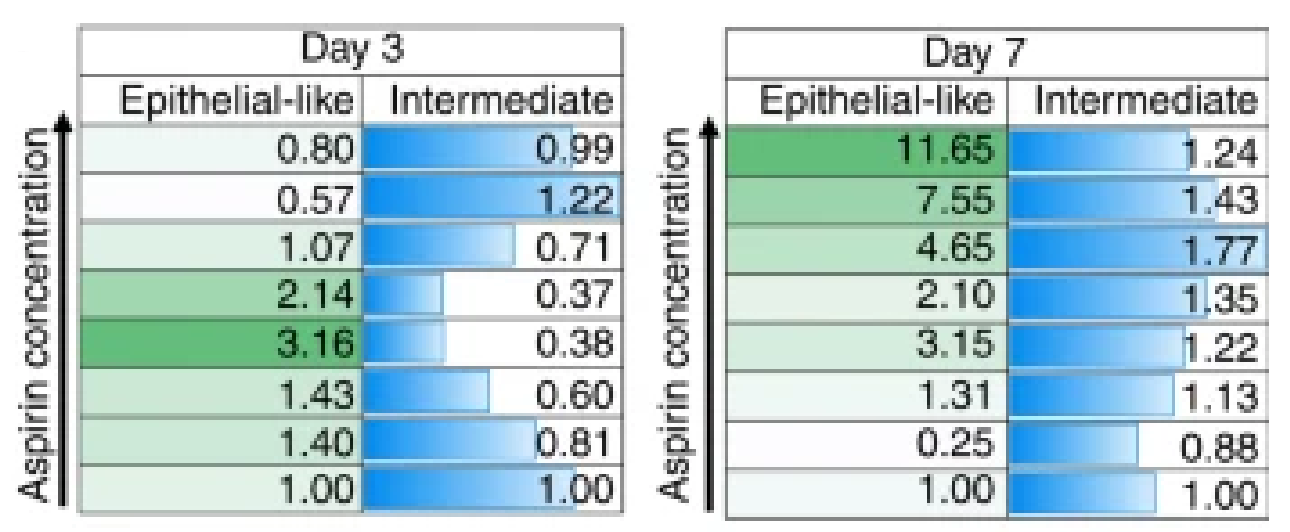

Immunostaining for CK and Vim

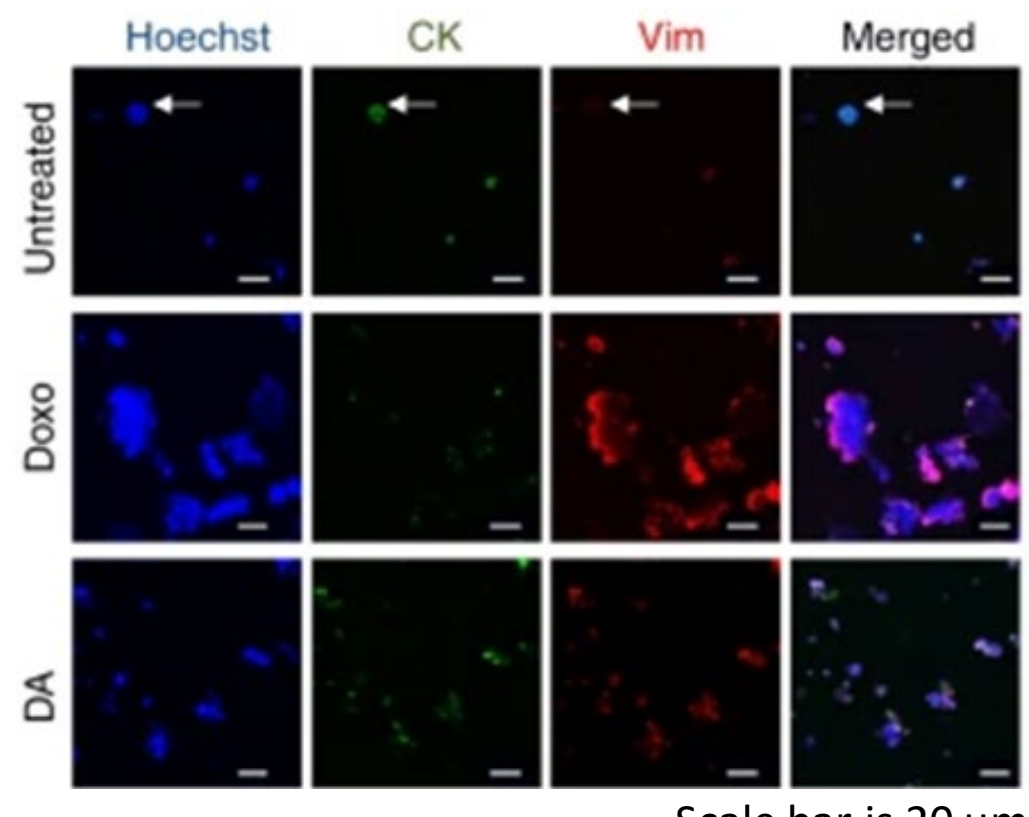

Scale bar is $20 \mu \mathrm{m}$ 


\section{Discussion}

- Compared with the treatment with doxorubicin alone, the intracellular oxidative activity in the sample under combinatorial DA treatment was reduced, as demonstrated by the intensity of Calcein AM. We demonstrated that the treatment outcomes were mediated by the reduction of COX-2, which was associated with inflammation triggered by ROS.

- Overall, the preclinical model could be used as a proof of concept to demonstrate the efficacy of antiinflammatory combinatorial therapies by influencing oxidative stress. Similar research could provide a basis for more DNA-related cancer treatment research in the future. 


\section{Acknowledgements}

Thanks to our lab members:

KHOO Bee Luan (Dr)

ZHANG Jing

DENG Yanlin

LIAO Junchen

FU Yatian

LI Wei

ZOU Shangjie

CHEN Chun kwan

WANG Shan

GUO Wang

Microsystems for Personalised Medicine (MPM) Lab

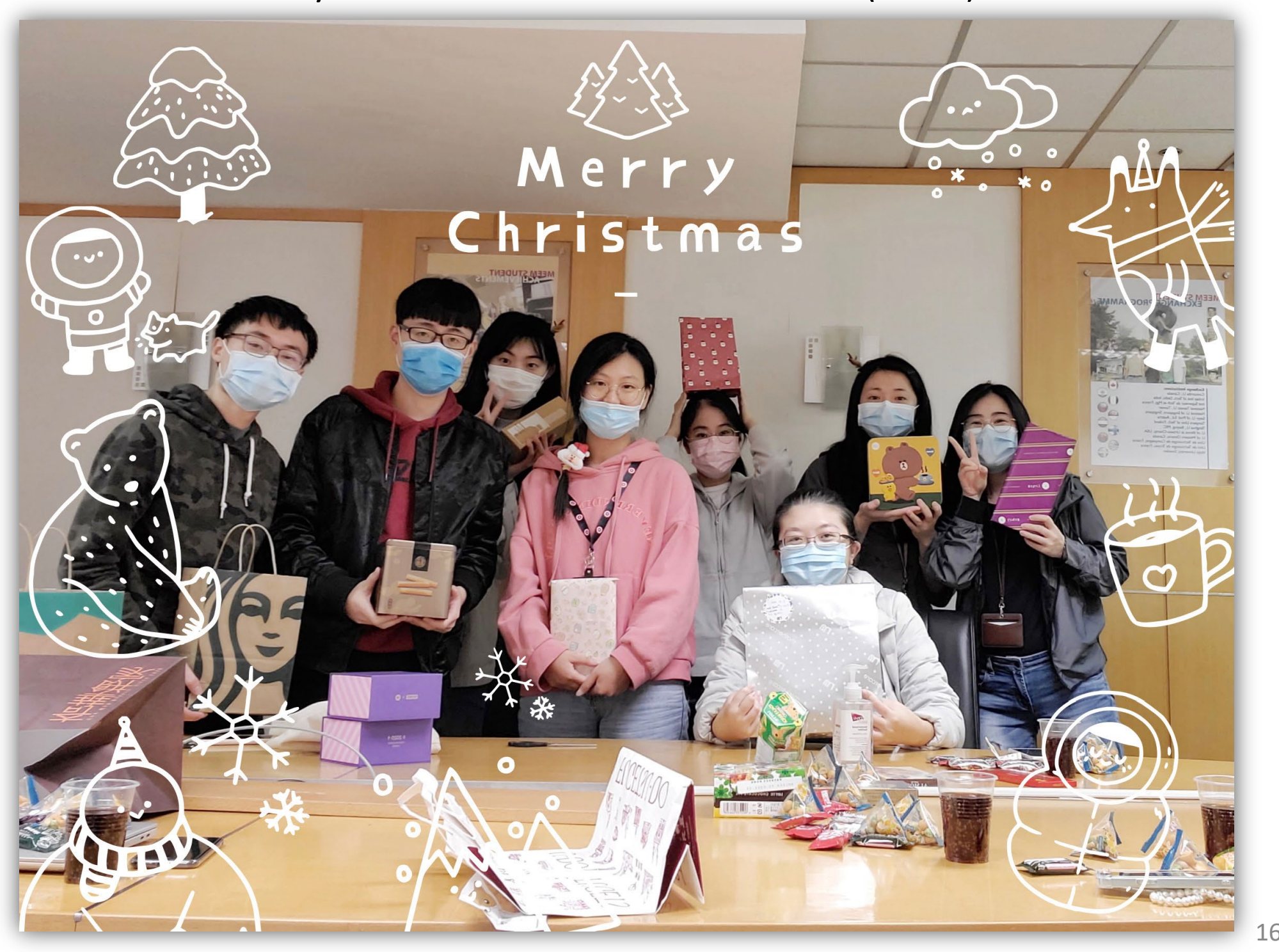




\section{Thank you!}

